\title{
Improved Technology of Furrow Irrigation on Mountain Slope Fields
}

\author{
Nurlibay T. Manabaev ${ }^{1}$, Farida H. Aubakirova ${ }^{1}$, Gulmira S. Kenzhibaeva ${ }^{1}$, Roza O. Zhumabaeva ${ }^{1}$ \\ \& Bauyrzhan Zh. Assylbekov ${ }^{1}$ \\ ${ }^{1}$ South-Kazakhstan State University named by M. Auezov, Shimkent, Kazakhstan \\ Correspondence: Nurlibay Manabaev, South-Kazakhstan State University named by M. Auezov, Shimkent, \\ Kazakhstan. Tel: 7-705-950-5436. E-mail: nmanabaev@mail.ru
}

Received: May 15, 2015 Accepted: June 16, 2015 Online Published: August 15, 2015

doi:10.5539/jas.v7n9p182 URL: http://dx.doi.org/10.5539/jas.v7n9p182

\begin{abstract}
The article reviewed furrow irrigation of agricultural crops, as the most common method of surface irrigation, given its advantages and disadvantages. It is noted that in order to achieve uniform wetting of the soil water flow fed into the furrows is increased, while the excess water is collected at the end of the furrow, and then reset the bleed water collection network. The dependence of uniform wetting of the field on the length of the furrow, the water flow, soil fertility and slope areas. Noticed, that the loss of water in the discharge of up to $40 \%$ of the irrigation norms, especially characteristic fields slopes steep. It is noticed that the loss of water in the discharge of up to $40 \%$ of the irrigation norms, especially characteristic for fields with steep slopes. Blow-off water washes away the fertile soil from fertilizers applied to improve soil fertility and crop yields. It is shown that as a result of irrigation erosion are reduced not only agricultural yields, but also quality of products.
\end{abstract}

Keeping traditions furrow's watering, ubiquitous in the Central Asian region, we have identified an opportunity to improve the technology of furrow irrigation on slop of the mountains fields, the strengths of which are water conservation, the preservation of fertility and other soil properties, the relevant environmental requirements.

At the cutting on the three-wheeled tractor furrows his rear wheel compared to the front more than compacted soil between rows, furrows and the soil located between the wheels, it is not compacted. For this reason, the water permeability of the soil of different grooves will vary accordingly the velocity of the water in the furrows, and the time until the end of the down hill furrow different. To address this shortcoming, it proposed to equip the new cultivator working organization in the form of additional wheels with different pitches density pattern on the tires, which will be left in the furrows respectively different path for the movement of water when watering. Irrigated water, moving the ejected trace left working organization will be overcome by a different path length in the furrows, but in the end comes at the same time different grooves. This allows the soil furrows evenly moistened, to the same start time of water in the furrows reduced accordingly reduced the feed water flow. The volume of waste water is also reduced, which prevents erosion of the soil. The advantages of this technology furrow irrigation on fields with large slopes.

Keywords: furrow, uniformity of soil moisture, erosion, working organization, step density pattern

\section{Introduction}

On irrigated land, depending on the crop, environmental conditions, agricultural technology and economic requirements apply various types of irrigation: vegetative, pre, before planting, after planting, water loading, washings and others. Carry them in different ways, each of which corresponds to a certain way of watering. So, in wide row crops watered one of the simplest and most affordable ways to soil moisture - furrows. With this irrigation water is distributed over the surface of a flow field in and blunt furrow and into the soil, mainly in the lateral direction, i.e. capillary action. It should be noted that the process of water absorption of the grooves is difficult, because soil layers have different water permeability. Soaking in the slopes of the furrow is more intense than the bottom: in sandy soils $-1.2 \ldots 1.7$ times; in $-1.75 \ldots 2.5$ times. This is due to greater freedom of action of capillary forces and independent outflow of air from the soil away from the groove (Markov, 1981).

Widespread furrow irrigation due to its following advantages:

> Under furrow irrigation occurs capillary wetting of the soil water, which better preserves its lumpy structure; 
$>$ Loosening the soil is allowed, providing aeration and improved flow nitrification process;

$>$ There is a possibility of watering required irrigation rates;

$>$ Reduced water loss through evaporation.

Nevertheless, furrow irrigation has disadvantages, among which are substantial water loss through discharge in the fields with steep slopes and tight soils; low coefficient of water use; uneven moistening of irrigated fields.

Research scientists (Ermaganbet, Dzhakeshov, \& Imanbaev, 2011) show the following distribution of water losses in the existing technology of furrow irrigation sprinklers time:

$>$ Filtration and evaporation of 15 ... 20\% percent;

$>\quad$ Discharge, leaks and evaporation depth of $20 \ldots 40 \%$, including due to non-simultaneous distribution to the front watering up to $8 \%$, pre-irrigation for organization $5 \%$ percent reasons.

At the same time, we must bear in mind that in the countries of Central Asia every year more sharply raises the question of water scarcity and the issue of careful attitude to water is now particularly relevant.

The working hypothesis of this paper is that the uniformity of soil moisture in the furrow on mountain slope fields is achieved by adjusting the path of the irrigation water in the furrow, depending on the density of the soil.

At the same time the following tasks:

$>$ Improving technology furrow's irrigation in areas with steep slopes through the development of new working organization of the cultivator;

$>$ Reduction of waste water for irrigation and control the path of the water in the furrows;

$>$ Preservation of soil fertility and other soil properties, the relevant environmental requirements due to reduced erosion, irrigation erosion.

\section{Methods}

Carried out Experiments in 2003-2004 years. The objects of research are the fields with medium and large slopes at sowing of cotton with $60 \times 60 \mathrm{~cm}$ and $90 \times 90 \mathrm{~cm}$ aisles. Studies used the principles of the system analysis and action in land reclamation techniques (field, isometric, laboratory).

\section{Results}

At present time under furrow irrigation, with an average slope field of the order $i=0.002 \ldots 0.01$ and deviation greater than the average $\mathrm{i}>0.01$ (slope of the mountains fields) compliance with the above requirements causes great difficulties. In fields with steep slopes are not planting scheme with spacing $90 \mathrm{~cm}$ of soil moisture due to the uneven furrows and the sharp increase in costs and duration of irrigation water. On such steep gradients recommended planting crops with row spacing of $60 \mathrm{~cm}$ or $60 \times 30 \mathrm{~cm}$ scheme (Figure 1). 


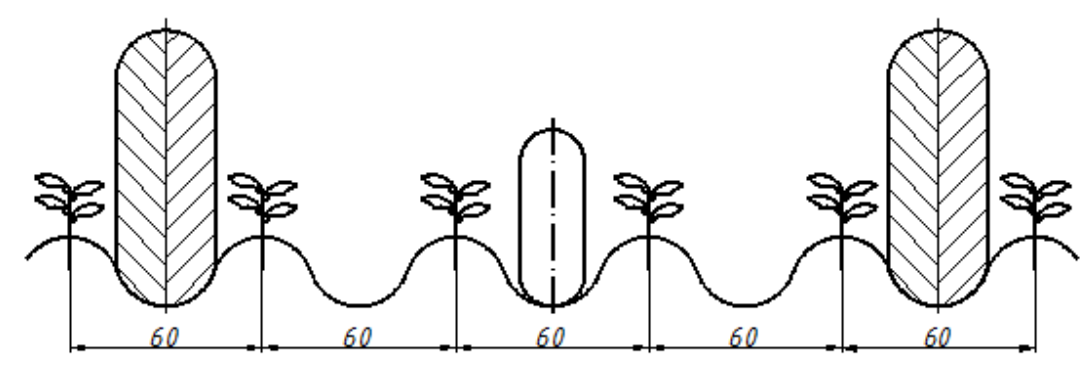

(1)

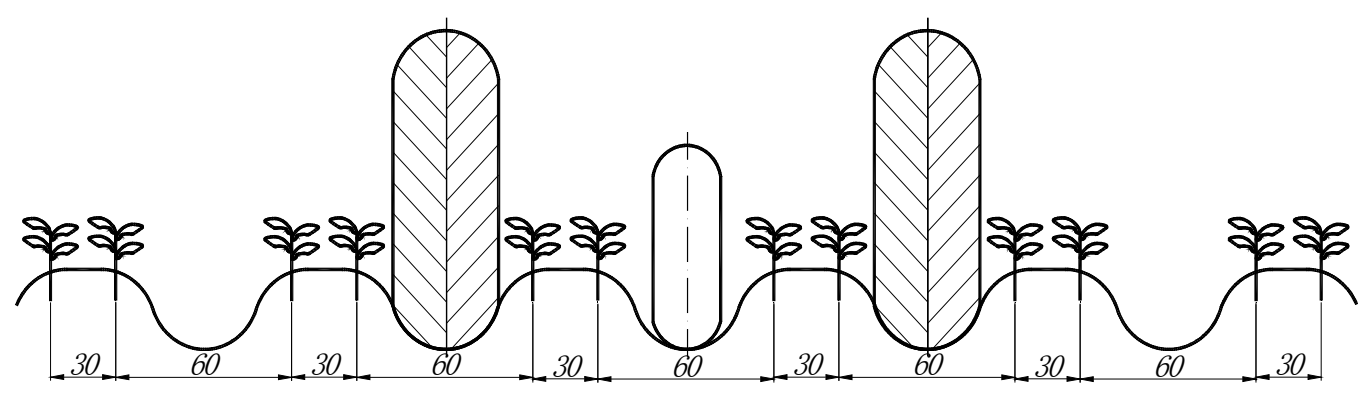

(2)

Figure 1. Schematic placement wheels tillage machine

Note. 1) Spacing $60 \mathrm{~cm}$; 2) Spacing $60 \times 30 \mathrm{~cm}$.

As seen in Figure 1, when the three-wheeled tractor on the rear wheel it over to the front more than compacted soil between rows, furrows and the soil located between the wheels, it is not compacted. For this reason, the water permeability of the soil of different grooves will vary accordingly the velocity of the water in the furrows, and the time lag of the jet at the end of the different furrow.

To eliminate abovementioned deficiencies identified the need to improve the processing unit through the development of new working organization. According to the research, as well as according to the practical data, to achieve uniform wetting of the soil under irrigation furrows of the field we offer the following field experimental working (Figure 2).

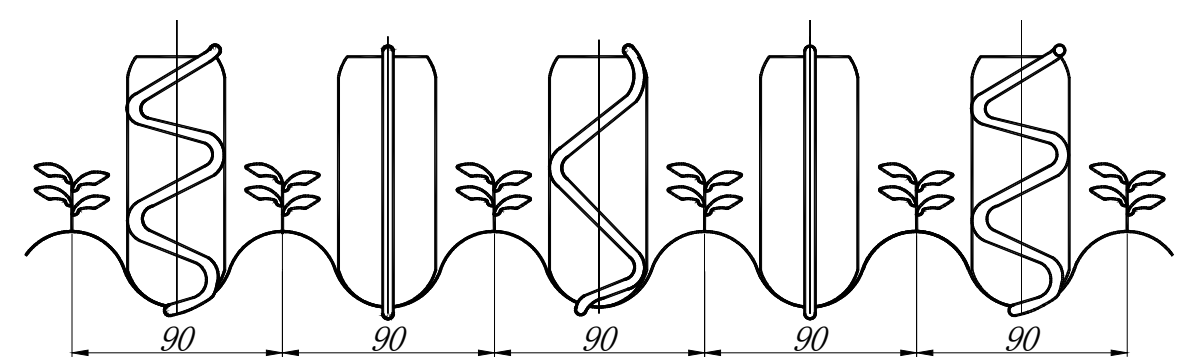

Figure 2. Scheme placement of experimental working body in the furrows

Recommendation construction for working organization, replacing hillers, is very simple and does not require complex operations. It is a wheel, dressed in cast rubber with embossed pattern (zigzag pattern) which is propagated in the furrow will leave a trail. Moreover, the wheels will have a different density pattern on the rubber. If the figure highlight the step (a zigzag pattern), the rubber wheels will run a three-step pattern density:

$>\quad$ Fold step (without zigzag straight line);

$>\quad$ With a 1.5-fold step (a rare zigzag pattern); 


\section{$>\quad$ With a 2-fold increments (frequent zigzag pattern).}

The unit is equipped with five additional wheels, which are arranged along its width. When passing the machine on the field will be held in each furrow wheel, which will be left in the furrows, respectively, three types of track. Two extreme wheel, passing the path of the tractor's rear wheels will have a 2-fold step-density pattern on the tires, because the soil these furrows more compacted under the action of the rear wheels of the tractor. The furrow, where the front wheel of the tractor, mounted additional wheel with a 1.5 -fold step because less soil compaction of the furrow. In the intermediate grooves that do not pass the tractor wheels, mounted wheels with fold step-density pattern, which leave a trace in the form of a straight line.

According to the proposed irrigation technology on the slope of the mountain field's water is simultaneously supplied to all furrows. However, it will move along the path trace the furrows left by additional rubber wheels. So, on the trail left by a wheel with a 2-fold step-density pattern, the jet will move for a long time because her way of artificially lengthened; on the trail of a wheel with a 1.5-fold step-density pattern of water path is shorter and the shortest route will be on the trail of a wheel with 1-fold step-density pattern. Thus, irrigated water, moving the ejected trace left of the additional wheels, will overcome a different way in the furrows, but in the end comes at the same time different grooves. This allows the soil furrows evenly moistened, to the same start time of water in the furrows decrease correspondingly reduced and supplied to the irrigation water consumption. In addition, the discharge of water is reduced, with even the appearance of waste water can be determined as reduced soil erosion, irrigation between 3 or 4 hours watering, as a result of absorption of moisture in the furrow, soil erosion stops completely.

Considering results cultivator with a new working body, its benefits:

$>$ Soil furrows evenly moistened;

$>\quad$ Furrow not crumble and earth fissures, as well as application of fertilizers are not washed away;

$>\quad$ The use of different density steps drawing on the additional molded rubber wheels leave traces in the furrows, reduces the accumulation of waste water in the furrow;

$>$ Irrigation water supplied to simultaneously reaches the end of grooves, which reduces the flow rate and the long time of irrigation;

$>$ Provides full saturation of water plants, which leads to a good harvest.

\section{Discussion}

The uniformity of distribution of irrigation standards along the length of the furrow length of $100 \ldots 200 \mathrm{~m}$, ranges $0.5 \ldots 0.7$, which leads to loss of crops from 2 to $15 \%$ of the non irrigation square. When watering the long furrows $(300 \ldots 400 \mathrm{~m})$, the coefficient of uniformity of soil moisture takes even lower values (Bezborozdov, 2010). The main purpose of rational use of water resources scientists from different countries being sought more productive and economical use of irrigation water. So, for uniform distribution of water in the furrows, and increase productivity when watering I. L. Bezuevsky and A. V. Shuravilin suggest the use of mobile irrigation units PAP-165 and PAP-165U. Watering is carried by the transverse scheme using flexible pipelines, with changeable waters productivity increases to $2 \ldots 3$ hectares (Olgarenko, 2011). Usually, in order to achieve uniform wetting of the soil water flow fed into the furrows is increased, i.e. that the jet ran until the end of the furrow served increased water consumption. This excess water is collected at the end of the furrow, and then reset water collection and relief lines. For reduce surface discharge when watering academician B.A.Shumakov's was suggest the possibility and development of a variable irrigation jet (Y. G. Bezborozdov \& A. G. Bezborozdov, 1998). In the furrow is supplied to the initial flow rate until the spray does not run so far to the end of the furrow. Then the flow is reduced, and in order not to complicate the process of watering, the regulated flow is fed into a furrow to wet the soil furrow.

According to research scientists, by controlling the flow of water supplied to the groove, reducing the amount of waste water and the soil furrows evenly moistened. However, it should be noted that the duration of watering is increased. To moisten the labia rules on tight soils with enough aligned surface used furrow irrigation-slots. From conventional furrow, they differ in that below the bottom of the furrow cut into the narrow slit of width 3.5 $\mathrm{cm}$, a depth of about $17 \mathrm{~cm}$, then the total depth of the furrows with slots increases and is $35 \ldots 40 \mathrm{~cm}$. Wetted perimeter of such furrows as compared to conventional grooves is increased by 2.5 times, so the water permeability of the soil increased significantly. The furrows cut into half-slot Wall chasers - Sheller, the water flow in the groove is $2 \ldots 2.5 \mathrm{l} / \mathrm{s}$, productivity is increased while irrigators.

Practice has shown that in this case the coefficient of uniformity of moisture rises to $0.8 \ldots 0.9$, and loss of flow 
to discharge excess water account for $5 \%$ of the initial flow (Bezborozdov, 2010). According to research by furrow irrigation, in the upper part having a parabolic section with a roll on the bottom, and at the bottom - the keystone without stitching, uniformity coefficient of irrigation norm with downhill jet to an average of 0.4 . To improve the uniformity coefficient of 0.8 to 0.7 glaze conducted with considerable discharge surface, in this case, the water release was more than $15 \ldots 20 \%$.

In Australia, the experimental tests conducted by furrow irrigation of cotton. The study found that an increase in the duration of watering from 4 to 16 hours aeration of the soil deteriorates as a consequence of fiber yield per unit area is reduced to $8 \%$ due to reduction in number of bolls. Referring to the results, the authors conclude that the reduction in the duration of watering leads to improved soil aeration, thereby increasing the yield of cotton (Akhmedzhanov \& Manabaev, 1992).

With a lack of the water in plants hydrolysis processes predominate over the processes of synthesis, sharply reduced transpiration and assimilation, there are losses of organic matter. Therefore, proper preparation of the soil for the cultivation of plants, proper care for them during the growing season, timely pest control, control of soil fertility needed for the cultivation of crops, irrigation regime observance of features - it is the event, without which the technology intensive cultivation of crops, which leads to high rates.

It is worth noting that even if the irrigation level field without much slope, there is a lot of problems when watering. Accordingly, the problems associated with the organization of irrigation land with an average slope or large, and growing even more complicated. Considering that full irrigation of plants in the area is a separate problematic task on slope of the mountains fields, you can see how much this process is complicated if at the same time take into account the problem of erosion of soil by watering.

According to the observations made by M. N. Zaslavsky (Khamidov et al., 1987), enhancing the degree of erosion of the soil under irrigation adversely affects the yield of crops. So, in the lands where soil erosion is insignificant, the yield falls to $10 \ldots 20 \%$, with an average erosion of soil $-40 \ldots 60 \%$, with strong $-60 \ldots 80 \%$.

Simultaneously, on soils exposed to erosion, and reduced crop quality products. All this affects the increase in production costs and leads to a reduction of profits.

On slope of the mountains fields Irrigation soil erosion causes considerable damage to cotton yield. Its emergence as a result of irrigation can be recognized mainly in the following characteristic features:

$>$ Slopes furrows (left or right), the most vertical;

$>\quad$ An increasing number of waste water in the farm irrigation systems;

$>\quad$ Changes the properties of the soil and its horizons, for example, other structural properties of the soil.

Each every year, as a result of irrigation erosion per hectare of irrigated land is eroded by an average of 100 tons of fertile soil. Moreover, the soil is washed away not only as a physical organization, but with her blurred useful substances in its composition: about $100 \mathrm{~kg}$ of nitrogen, $115 \mathrm{~kg}$ of phosphorus and other elements. If we take into account that in addition to these elements in the soil, there are other valuable nutrients that are essential for crop improvement, it is not difficult to calculate the extent of economic damage from erosion.

Necessary note because of the erosion of soil fertility drops sharply, it leads to an acceleration of its aridity. Therefore, in areas prone to erosion, provides for measures to combat erosion and improve soil fertility, which are among the first tasks in the application of soil conservation farming systems.

The question of optimal row spacing and length of the grooves in growing cotton has always attracted the attention of researchers. The results of the research organizations and the experience of the farmers indicated that the sowing of cotton in the fields of the middle and low slopes does not give good results. The reason is that for the irrigation of fields using deep furrows, uniform wetting which is very complex. Therefore, to improve the intensive cotton production, increasing yields of irrigated fields must be separately taken into account the composition of the soil, its fertility and slope of the field.

The observations and practice of business executives showed that lands with small and medium slopes are widely sown row cotton in reality has some advantages. The results shows in Table 1 in wide sowing of cotton in the fields Pahtachinsk district of Samarkand region, where it is possible to trace that the additional average cotton yield was more than 2.6 hundredweights in one hectare. 
Table 1. Results in wide cotton planting

\begin{tabular}{|c|c|c|c|c|c|c|}
\hline \multirow{3}{*}{$\mathrm{N}$ site field } & \multicolumn{3}{|c|}{ Square of sown field } & \multirow{2}{*}{\multicolumn{2}{|c|}{$\begin{array}{c}\text { Yields of cotton } \\
\text { To spacing }\end{array}$}} & \multirow{3}{*}{$\begin{array}{l}\text { Additional yield from } 1 \text { ha, } \\
\text { hundredweight }\end{array}$} \\
\hline & \multirow{2}{*}{ Total indicates } & \multicolumn{2}{|c|}{ Including spacing } & & & \\
\hline & & $60 \mathrm{~cm}$ & $90 \mathrm{~cm}$ & $60 \mathrm{~cm}$ & $90 \mathrm{~cm}$ & \\
\hline N1 & 1528 & 970 & 558 & 34,2 & 38,3 & $+4,1$ \\
\hline $\mathrm{N} 2$ & 1591 & 313 & 1278 & 38,7 & 40,4 & $+1,7$ \\
\hline N3 & 1780 & 1366 & 414 & 41,7 & 43,04 & $+1,3$ \\
\hline N4 & 1165 & 563 & 602 & 46,8 & 47,8 & $+1,0$ \\
\hline N5 & 1743 & 969 & 774 & 39,6 & 44,3 & $+4,7$ \\
\hline Average & & & & 40,2 & 42,8 & $+2,6$ \\
\hline
\end{tabular}

As shown by the economic calculations of profitability in growing cotton in wide sowing of cotton compared with closed drills is increased by $29 \ldots 35 \%$ and labor costs reduced by $19 \ldots 20 \%$. However, in the fields with steep slopes widely sown planting cotton has led to a decrease in its productivity, which is confirmed by multiply research (Khamidov et al., 1987) conducted in the field. For example, to research according authors Sokolov (1968) at wide-crop cotton at high surface slopes sharply reduced yields; Research of Medis and Nosirova (1966) showed a decrease in yield of 3.7 ... 13.3 quintals per hectare; Experiences of Kabaeva and Shalashilinova (1965), Kashkarova (1966), and Nadirmatova (1966) did not give the expected yield of cotton. Reduced yields according to researchers due to two factors: first, the presence of the slope area; secondly, uneven soil moisture during irrigation furrows. In this case, the occurrence of irrigation erosion resulted in lower crop yields. The fields, consisting of sections with medium and large slopes, with wide-row sowing of cotton irrigated by deep furrows through which water is absorbed into the compacted soil. There is erosion of slopes and bottom of the grooves, in some cases, soil erosion reaches the recesses $15 \ldots 20 \mathrm{~cm}$. In these areas irrigated water penetrates into the deeper layers of the soil, the soil is not saturated with moisture aisles (Bezborozdov, 2005). This increases the amount of waste water during the growing season and on fields with slopes such soil erosion reaches $240 \ldots 250$ tons per hectare. This again emphasizes that the erosion of the soil not only affect the water flow and the length of the grooves, but also to a large extent the slope terrain.

\section{Conclusion}

In view of above, under furrow irrigation in the fields with steep slopes (slope of the mountains fields) in a production environment it is recommended to use the cultivator equipped proposed working body in the form of additional wheels with different pitches density pattern on the rubber. It should also be noted that by using the technology of irrigation furrow's an opportunity for the traditional planting scheme with spacing $90 \mathrm{~cm}$, which improves the phytosanitary condition of landings and increases the efficiency of use of agricultural machinery.

\section{References}

Akhmedzhanov, M. A., \& Manabaev, N. T. (1992). Alignment temporary sprinkler. Journal of Agriculture of Uzbekistan, 8, 47-49.

Bezborozdov, Y. G. (2005). Energy, environmental and economic efficiency of water-saving irrigation technologies. Journal of Agricultural Sciences, 6, 65-67.

Bezborozdov, Y. G. (2010). Theoretical study and practical implementation of irrigation for row crops shielded furrows (Doctoral dissertation). Russian Academy of Agricultural Sciences, All-Russian Research Institute of Hydraulic Engineering and Land Reclamation named by A. N. Kostyakov, Moscow, Russia.

Bezborozdov, Y. G., \& Bezborozdov, A. G. (1998). The method of reducing the loss of irrigation water. Journal of Agricultural Sciences, 3, 32-36.

Ermaganbet, A. Z., Dzhakeshov, K. S., \& Imanbaev, K. K. (2011). Irrigated agriculture and their analysis in the Republic of Kazakhstan. Proceedings of the 7th International Scientific and Practical Conference. Almaty, Kazakhstan: Kazakh National Agrarian University.

Khamidov, H., et al. (1987). Increase of fertility of soil erosion. Tashkent, Tashkent Mehnat.

Markov, E. S. (1981). Agricultural hydraulic reclamation. Moscow: Kolos.

Olgarenko, G. V. (2011). The strategy of the scientific and technical work on the development of new techniques 
for irrigation in the development program of land reclamation. Irrigation and Water Management, 6, 5-8.

\section{Copyrights}

Copyright for this article is retained by the author(s), with first publication rights granted to the journal.

This is an open-access article distributed under the terms and conditions of the Creative Commons Attribution license (http://creativecommons.org/licenses/by/3.0/). 